\title{
Programa em práticas educativas positivas para educadores sociais de instituições de
} acolhimento: Estudo de viabilidade

\author{
Positive educational practices program for social educators of out-of-home care: Feasibility study \\ Programa en prácticas educativas positivas para educadores sociales de intituciones de acogida:
}

\author{
Estudio de viabilidad
}

Recebido: 19/07/2021 | Revisado: 24/07/2021 | Aceito: 25/07/2021 | Publicado: 01/08/2021

\author{
Bruna Wendt \\ ORCID: https://orcid.org/0000-0002-4625-2700 \\ Universidade Federal do Rio Grande do Sul, Brasil \\ E-mail: brunawendt@yahoo.com.br \\ Débora Dalbosco Dell'Aglio \\ ORCID: https://orcid.org/0000-0003-0149-6450 \\ Universidade Federal do Rio Grande do Sul, Brasil \\ Universidade La Salle, Brasil \\ E-mail: dddellaglio@gmail.com
}

\begin{abstract}
Resumo
O educador social configura-se como a principal referência protetiva e afetiva de crianças e adolescentes acolhidos. É fundamental, portanto, que estes profissionais sejam continuamente qualificados. O objetivo deste estudo foi avaliar a viabilidade do Programa Cuida, programa em práticas educativas positivas destinado a educadores sociais de instituições de acolhimento. O programa é composto de oito sessões que trabalham temáticas como regulação emocional, comunicação assertiva, estratégias de resolução de conflitos e autocuidado. O programa foi aplicado em dois grupos, totalizando 12 educadores sociais e os critérios de viabilidade analisados foram: Aceitabilidade/adesão; Avaliação do moderador pelos observadores; Satisfação dos participantes com o programa e com o moderador; Compreensão/generalização dos conteúdos. Foram realizadas análises estatísticas descritivas e uma análise qualitativa a partir dos registros dos observadores, participantes e moderador. Os resultados obtidos foram satisfatórios considerando os critérios avaliados e as mudanças introduzidas no programa são apresentadas. Este estudo demonstra a viabilidade da proposta de intervenção para que siga para estudo de eficácia.
\end{abstract}

Palavras-chave: Acolhimento institucional; Educador social; Práticas educativas; Viabilidade.

\begin{abstract}
The social educator is the main protective and affective reference for children and adolescents in care. It is essential, therefore, that these professionals are continuously qualified. The objective of this study was to evaluate the viability of Programa Cuida, a program to improve the positive educational practices for social educators in residential care. The program consists of eight-weekly sessions that work on topics such as assertive communication, conflict resolution strategies, emotional self-regulation and self-care practices. The program was applied in two groups, totaling 12 social educators and the feasibility criteria analyzed were: Acceptability/adherence; Observers' evaluation of the moderator; Participants' satisfaction with the Program and moderator; Understanding/generalization of contents. Descriptive statistics analyzes and a qualitative data analysis plan were used, based on the records of observers, participants and moderator. The results obtained were satisfactory considering the evaluated criteria and the changes introduced in the program are presented. This study demonstrates the viability of the proposal to proceed to efficacy trial.
\end{abstract}

Keywords: Out-of-home care; Social educator; Educational practices; Feasibility.

\section{Resumen}

El educador social es la principal referencia protectora y afectiva para niños y adolescentes acogidos. Es esencial, por lo tanto, que estos profesionales estén continuamente calificados. El objetivo de este estudio fue evaluar la viabilidad del Programa Cuida, un programa de prácticas educativas positivas dirigido a educadores sociales de instituciones de acogida. El programa consta de ocho sesiones que trabajan sobre temas como la regulación emocional, la comunicación asertiva, las estrategias de resolución de conflictos y el autocuidado. El programa se aplicó en dos grupos, totalizando 12 educadores sociales y los criterios de viabilidad analizados fueron: Aceptabilidad/adhesión; Evaluación del moderador por los observadores; Satisfacción de los participantes con el programa y el moderador; Comprensión/generalización de los contenidos. Se realizaron análisis estadísticos descriptivos y un análisis cualitativo basado en los registros de observadores, participantes y moderador. Los resultados obtenidos fueron satisfactorios 
considerando los criterios evaluados y se presentan los cambios introducidos en el programa. Este estudio demuestra la viabilidad de la propuesta de intervención para proceder a un estudio de eficacia.

Palabras clave: Acogimiento institucional; Educador social; Prácticas educativas; Viabilidad.

\section{Introdução}

O acolhimento institucional é uma medida de proteção provisória e excepcional destinada a crianças e adolescentes cujos direitos foram ameaçados ou violados, não implicando privação de liberdade (Brasil, 1990). Embora esta medida tenha um caráter excepcional e provisório, as instituições de acolhimento ainda recebem um alto número de acolhidos, cerca de 34.157 crianças e adolescentes (Conselho Nacional de Justiça, 2020).

Neste contexto de cuidado integral, o educador social configura-se como a principal referência protetiva e afetiva dos acolhidos (Kappler \& Mendes, 2019; Marzol, Bonafé, \& Yunes, 2012), responsabilizando-se pelas suas necessidades básicas, como a higiene e a alimentação, e também pelos cuidados emocionais e psicológicos, ainda que os vínculos estabelecidos sejam temporários. A qualidade da vinculação e da interação entre educador social e acolhido apresenta-se como um importante fator de proteção nesse contexto (Silveira, Maruschi, \& Bazon, 2012).

A importância deste profissional e de suas práticas gera, para a instituição de acolhimento e órgãos gestores, a demanda de cuidá-lo e capacitá-lo continuamente para lidar com as complexidades que se apresentam. Por vezes, a falta de suporte institucional direcionado ao educador social reflete-se em discursos e ações marcados por frustrações e sentimentos de insegurança, impotência e insatisfação (Avoglia, Silva, \& Mattos, 2012). Também pode se relacionar a sintomas como a insônia, fadiga, irritabilidade, tristeza, pouca concentração e queixas somáticas, os quais, embora não preencham os requisitos para diagnósticos de transtorno mental, classificados pelo CID-10 ou DSM-V, podem produzir incapacidades funcionais e laborais (Romero, Akiba, Dias, \& Serafim, 2016).

Estudos vêm indicando a necessidade de implementação de uma política de recursos humanos que envolva formação inicial, capacitação continuada, supervisões de casos, incentivos e valorização destes profissionais (Barros \& Naiff, 2015; Guerra \& Del Prette, 2018; Moré \& Sperancetta, 2010). Além de ser oferecido um espaço de escuta e suporte, as trocas entre os educadores sociais favorecem os processos de identificação e de construção coletiva de estratégias de enfrentamento dos desafios cotidianos. Esse amparo técnico e afetivo é fundamental para que se rompa com a lógica assistencialista e, consequentemente, para maior qualificação e profissionalização da atividade.

São poucos os estudos brasileiros que apresentam propostas de intervenção com educadores sociais no contexto do acolhimento institucional. Um número ainda menor de pesquisas se propõe a avaliar as intervenções implementadas, a partir de critérios metodológicos, com o uso de instrumentos de medida contínua ou de pré e pós-intervenção (Bersch, 2017; Biscouto, 2012; Prada, 2007).

Bersch (2017) desenvolveu e implementou ao longo de seu doutorado um programa de formação para educadores sociais em um município do Rio Grande do Sul a partir da perspectiva teórico-metodológica da Abordagem Bioecológica do Desenvolvimento Humano, articulada com conceitos da Educação Ambiental. O Programa contou com 12 encontros presenciais de duas horas, e teve o intuito de potencializar a resiliência profissional dos trabalhadores e promover melhorias no espaço institucional. A partir da triangulação dos dados foi avaliado o impacto do processo formativo nas práticas educativas dos profissionais. A autora concluiu que os participantes melhoraram as suas capacidades de comunicação interpessoal, procurando interagir de forma mais bem humorada e afetiva com seus pares, demonstraram maior credibilidade no trabalho em equipe e interesse pelo trabalho dos colegas. Além disso, puderam refletir sobre novos projetos de futuro para os acolhidos e compreenderam a importância das atividades lúdicas e da linguagem corporal nas interações. O detalhamento dos objetivos, metodologia e procedimentos do programa encontram-se manualizados e disponíveis online (Bersch et al., 2019). 
Prada (2007) desenvolveu, implementou e avaliou um Programa de Treinamento em Práticas Educativas para monitores de abrigos para crianças vítimas de violência doméstica. O estudo tinha o intuito de investigar a qualidade da interação de 14 monitoras com 13 crianças ao longo de 12 sessões semanais, com duração de 90 minutos. Os resultados demonstraram que a frequência dos comportamentos de interação positiva das monitoras e das crianças (elogiar, brincar junto) aumentou, enquanto que a frequência dos comportamentos de interação coercitiva (repreensão, chantagens, olhares de desaprovação, punições) diminuiu após as sessões analisadas.

Biscouto (2012) propôs um programa de intervenção em práticas educativas semelhante para sete mães sociais de três instituições de acolhimento do Paraná. Foram realizados dez encontros, oito deles para a intervenção em grupo e dois para coletar as medidas de pré e pós-teste. Os resultados indicaram mudanças, embora discretas, nos padrões de interação entre profissionais e acolhidos, com aumento dos escores de práticas educativas positivas, como a monitoria positiva e o comportamento moral.

Compreende-se que é necessário ampliar as possibilidades teóricas e metodológicas para desenvolver novas propostas de capacitação, bem como utilizar critérios sistemáticos para avaliar os resultados. Dessa forma, tendo em vista a importância do desenvolvimento de intervenções direcionadas a profissionais que atuam em contextos de acolhimento, o objetivo deste estudo foi a avaliação da viabilidade do Programa Cuida, programa em práticas educativas positivas, a fim de verificar o potencial de a intervenção ser bem-sucedida e seguir para futuro estudo de eficácia-efetividade. Os estudos de viabilidade, embora não incluam em seu delineamento o teste de hipóteses, permitem que sejam traçados ajustes importantes para a intervenção principal subsequente.

\subsection{Descrição da intervenção: O Programa Cuida}

O Programa Cuida tem como objetivo principal proporcionar mecanismos de apoio educacional com vistas a melhorar/ampliar as habilidades e capacidades do educador social de instituições de acolhimento. O Programa é estruturado em oito encontros de duas horas, de modo que o primeiro e o último são direcionados, sobretudo, à aplicação de instrumentos de avaliação pré e pós-intervenção (T1 e T2) para investigar as evidências de eficácia do Programa.

A construção do Programa amparou-se teoricamente nos pressupostos da Parentalidade Positiva. Na Europa, os programas em Parentalidade Positiva direcionados a pais e a cuidadores têm crescido substancialmente com vistas a melhorar as habilidades parentais, reduzir o impacto dos fatores de risco e promover os fatores protetivos relacionados ao bem-estar físico, social e emocional das crianças (Rodrigo, 2016). Esses programas, recomendados e apoiados pelo Conselho Europeu desde 2006 (Council of Europe, 2006), demonstram a importância de intervenções precoces e preventivas para promover o desenvolvimento integral da criança e práticas de cuidado não violentas.

Sugere-se que os programas em parentalidade positiva sejam estruturados levando-se em consideração os seguintes princípios: (1) Vínculos afetivos protetores e estáveis; (2) Estrutura do contexto que proporcione orientação e supervisão; (3) Reconhecimento e valorização dos filhos; (4) Estimulação e apoio das aprendizagens; (5) Educação não violenta, excluindo-se todas as formas de castigo físico e/ou psicológico; e (6) Estimulação à autonomia, potencializando a percepção de que são agentes ativos, competentes e capazes. Tais princípios buscam trabalhar a afetividade, respeito, compreensão mútua, comunicação igualitária e busca de acordos (Martín-Quintana \& Rodrigo-López, 2013), a partir de um processo de mudança individual que possibilita uma reflexão e otimização do exercício da parentalidade (Rodrigo-López, Máiquez-Chavez, \& Martín-Quintana, 2010). Diversos estudos recentes vêm demonstrando que o uso de práticas parentais positivas está associado a melhores níveis de bem-estar emocional e a menor incidência de sintomas depressivos (Chen, Haines, Charlton, \& VanderWeele, 2019). Da mesma forma, tais práticas parecem funcionar como fatores de proteção frente a situações adversas na infância (Yamaoka \& Bard, 2019). 
No Programa Cuida foi utilizada como suporte metodológico para a condução dos grupos a Metodologia Experiencial (Martín-Quintana et al., 2009), também utilizada por Bersch (2017) em sua intervenção com educadores sociais. Tal metodologia não tem a pretensão de informar ou ensinar teorias prontas, nem de propagar verdades absolutas, ou receitas de “como fazer". Assim como a metodologia experiencial, as intervenções propostas pelo programa prezam pelo diálogo com os educadores sociais e pela construção conjunta, levando-se em consideração a bagagem e as experiências destes profissionais dentro do contexto institucional. É a partir desse conhecimento experiencial que as competências são fortalecidas (MáiquezChávez, Rodrigo-López, Capote, \& Vermaes, 2000), sobretudo quando se trata de competência no trabalho em grupos, os quais podem partilhar experiências comuns e singulares, como é o caso dos Educadores Sociais. Com isso, embora tenha sido utilizado um roteiro previamente estruturado para guiar a aplicação, os encontros não foram inteiramente padronizados, gerando reflexões e discussões distintas em cada grupo.

As sessões foram expositivas e vivenciais, teórico-práticas. As temáticas trabalhadas ao longo do Programa são associadas teoricamente ao desenvolvimento de práticas educativas positivas, como (1) Regulação Emocional; (2) Comunicação Assertiva; (3) Estratégias de Resolução de Conflitos; (4) Cuidado de si. Tais temáticas foram selecionadas com base em outros programas nacionais e internacionais em educação positiva de pais ou de educadores sociais (Bersch, 2017; Martínez-González, 2009; Prada, 2007; Silva, 2009).

A seguir, são apresentadas, resumidamente, as sessões, os objetivos, assim como as atividades e a "técnica da semana" referentes a cada encontro.

Sessão 1. Introdução e apresentações. Objetivo: Apresentações pessoais e apresentação do Programa Cuida; aplicação coletiva dos instrumentos (T1). Atividades: História do meu nome. Exposição dos objetivos e combinação das normas de funcionamento do Programa Cuida. Aplicação do Questionário Sociodemográfico e da "Escala de Competências Educativas, Emocionais e Sociais para Educadores Sociais” (Wendt \& Dell'Aglio, submetido) (T1).

Sessão 2. Conhecer para intervir. Objetivo: Refletir sobre o papel do educador social no contexto institucional e promover sensibilização sobre sua importância e responsabilidade neste espaço. Atividades: Discussão coletiva sobre a função do educador social. Atividade 1: Vídeo sobre o acolhimento institucional e sobre o papel do educador (elaborado pela pesquisadora); Atividade 2: "Maria é portuguesa, está visitando o Brasil e gostaria de saber o que faz um Educador Social. Conte a ela"; Atividade 3: Montagem de uma colcha de retalhos de papel colorido, propondo uma reflexão sobre a composição da rede de atendimento que dá suporte à instituição. Técnica da semana: Anotar três situações ocorridas em que a sua função como educador social foi essencial.

Sessão 3. Práticas Educativas Positivas. Objetivo: Evidenciar meios de implementar práticas educativas positivas e suas vantagens para o desenvolvimento do acolhido e para as interações na instituição. Atividades: Explanação sobre as práticas educativas positivas e as formas de implementá-las no contexto do acolhimento institucional. Diferenciação entre práticas indutivas e coercitivas e vantagens e desvantagens do seu uso; Atividade 1: Vídeo sobre práticas educativas positivas (elaborado pela pesquisadora); Atividade 2: Papel amassado (Schuler \& Dias, 2014). Atividade 3: Role-play: encenações em duplas. Técnica da semana: Anotar alguma situação em que tenha sido implementada uma prática educativa positiva.

Sessão 4. Regulação emocional. Objetivo: Auxiliar no reconhecimento e no desenvolvimento de habilidades e estratégias de regulação das próprias emoções para resolução mais adaptativa dos conflitos com os acolhidos. Atividades: Discussão inicial sobre o que são as emoções e a regulação emocional e sobre como melhorá-la. Diferenciação entre estratégias adaptativas e estratégias desadaptativas. Atividade 1: Vídeo sobre regulação emocional (elaborado pela pesquisadora); Atividade 2: Balão das emoções; Atividade 3: "Você reconhece essas emoções?": atividade com fotos; Técnica 1: Registro de pensamentos disfuncionais; Técnica 2: Relaxamento conduzido pelo moderador. Técnica da semana: Redação expressiva 
(Gortner, Rude, \& Pennebaker, 2006): escrever, todos os dias, durante cinco minutos, sobre as emoções geradas frente a situações negativas/conflitos.

Sessão 5. Comunicação assertiva. Objetivo: Auxiliar na promoção de habilidades de comunicação positivas e assertivas para melhorar as relações com os acolhidos e com colegas. Atividades: Apresentação dos diferentes tipos de comunicação: passiva, assertiva e agressiva. Como e porque comunicar-se assertivamente e a importância da escuta. Atividade 1: Vídeo sobre comunicação assertiva (elaborado pela pesquisadora); Atividade 2: "Jogo da mímica", para explorar outras maneiras de comunicar-se; Atividade 3: Role-play: encenação em duplas; Técnica da semana: Dar feedbacks positivos para o maior número de pessoas que puder e refletir sobre a reação delas.

Sessão 6. Estratégias de resolução de conflitos. Objetivo: Auxiliá-los a resolver conflitos de maneira positiva, implementando limites e consequências coerentes com a situação, sem fazer uso de práticas coercitivas. Atividades: Diferenciação entre estratégias positivas e negativas para a resolução de conflitos e ênfase na perspectiva colaborativa para resolução de conflitos. Atividade 1: Vídeo sobre estratégias de resolução de conflitos (elaborado pela pesquisadora); Atividade 2: Brainstorming ou Tempestade de ideias da palavra "conflito"; Atividade 3: A teia cooperativa; Atividade 4: Método de Exploração de Soluções. Técnica da semana: Listar alguma situação em que tenha utilizado estratégias positivas/construtivas de resolução de conflitos.

Sessão 7. Cuidado de si. Objetivo: Evidenciar a importância e estimular o desenvolvimento de estratégias de autocuidado no âmbito pessoal e profissional do educador social. Atividades: Refletir coletivamente sobre a importância do autocuidado para o bem-estar pessoal e profissional e de porquê preservar e compartilhar afetos positivos, como otimismo, esperança, gratidão. Atividade 1: Vídeo sobre cuidado de si (elaborado pela pesquisadora); Atividade 2: Quebra-cabeça do autocuidado. Atividade 3: “Como você tem cuidado de si?" (já faço/preciso fazer). Atividade 4: em duplas ou trios praticam "Fale sobre mim para mim". Técnica 1: Relaxamento conduzido pelo moderador. Técnica da semana: Qual é a qualidade/força que hoje se destacou em mim? Fazer isso durante todos os dias da semana.

Sessão 8. Encerramento e confraternização. Objetivo: Finalização da intervenção, reflexão sobre o que foi aprendido, aspectos positivos e negativos do programa. Atividades: Síntese dos aprendizados do programa e de sugestões para mudanças. Confraternização com os participantes (lanche coletivo); Reaplicação da escala (T2) e aplicação da Ficha de Satisfação com o Programa.

\section{Metodologia}

\subsection{Delineamento}

Este artigo apresenta o primeiro ensaio de modo estruturado do Programa Cuida, como estudo de viabilidade, a fim de verificar o potencial de a intervenção ser bem-sucedida e poder ser replicada em futuros estudos de eficácia. Entende-se o estudo de viabilidade como um recorte conduzido previamente a um estudo maior para ajustar e/ou conduzir retificações no delineamento e verificar se a intervenção tem condições/potencial para seguir para o ensaio de eficácia (National Institute For Health Research/NIHR, 2017). Ao contrário de um estudo de eficácia, o objetivo de um estudo de viabilidade não é testar hipóteses (Durgante \& Dell'Aglio, 2018). Embora não haja critérios metodológicos consensuais estabelecidos, seguiu-se as orientações de Bowen et al. (2009) e de Durgante e Dell’Aglio (2018), que sugerem áreas fundamentais a serem verificadas em estudos de viabilidade, que são as seguintes: (1) Aceitabilidade e adesão; (2) Avaliação do moderador pelos observadores (Habilidades Sociais e Integridade/fidelidade); (3) Satisfação dos participantes com o programa e com o moderador; (4) Compreensão/generalização dos conteúdos pelos participantes. Esses critérios foram investigados a partir de estatísticas descritivas e de um plano de análise qualitativa de dados. 


\subsection{Participantes}

A amostra não probabilística foi composta por 12 educadores sociais (11 mulheres, 1 homem), com idades entre $35 \mathrm{e}$ 59 anos $(X=50.25 ; D P=7.44)$, com variação de 5 a 33 anos de tempo de trabalho $(X=19.58 ; D P=10)$, divididos em dois grupos. Todos os educadores sociais eram concursados do estado do Rio Grande do Sul e trabalhavam em instituições de acolhimento de quatro regiões diferentes da cidade onde foi realizada a pesquisa. Os critérios de inclusão para compor a amostra foram: a) Ter, no mínimo, Ensino Médio concluído; b) Desempenhar a função de educador social em instituição de acolhimento de crianças e adolescentes há, pelos menos, 6 meses; c) Aceitar participar das avaliações antes (T1) e após o término do programa (T2).

Também participaram quatro observadoras previamente treinadas que se alternaram em duplas ao longo dos oito encontros de cada grupo. Os requisitos para os observadores foram: a) Ter recebido capacitação teórica relevante na área; b) Ter recebido treinamento sobre os procedimentos do programa e instrumentos de avaliação; c) Familiarizar-se com o roteiro do programa (objetivos e procedimentos das sessões); d) Participar de reuniões periódicas para avaliar o andamento do programa. Esses critérios buscaram assegurar uma maior qualidade metodológica (Levitt et al., 2018).

\subsection{Instrumentos}

Para avaliação dos critérios de viabilidade mencionados anteriormente, dados foram obtidos através de:

(1) Questionário Sociodemográfico (ANEXO J): para investigar informações dos participantes como sexo, idade, escolaridade, estado civil, tempo de profissão e cursos de capacitação anteriores.

(2) Ficha de avaliação do desempenho do moderador (adaptada de Durgante, 2019) (ANEXO K), preenchida por dois observadores treinados presentes em cada sessão. O questionário inclui questões sobre: habilidades sociais e integridade/fidelidade do(a) moderador(a) para com os processos na implementação da intervenção, adesão dos participantes às dinâmicas e técnicas sugeridas durante as sessões e três questões descritivas para relato de acontecimentos significativos, temas emergentes e outras observações.

(3) Ficha de avaliação de satisfação dos participantes (adaptada de Durgante, 2019) (ANEXO L): com questões em escala likert e questões abertas, para avaliar a satisfação com o programa, a clareza e compreensão dos conteúdos abordados e a generalização do aprendizado, após o término do programa. Esta ficha contém nove perguntas de autorrelato e cinco questões descritivas.

(4) Diário de campo do moderador para registros de observações, interpretações e inferências feitas ao término de cada sessão do programa. Os dados obtidos dão suporte qualitativo às avaliações dos observadores, como critério adicional para maior qualidade metodológica (Creswell, 2003).

\subsection{Procedimentos e considerações éticas}

O estudo foi aprovado pelo Comitê de Ética em Pesquisa da Universidade Federal do Rio Grande do Sul, sob o parecer 2.723.700 e seguiu as diretrizes e normas da Resolução $\mathrm{N}^{\circ}$ 510/2016 para regulamentação ética em pesquisas com seres humanos (Conselho Nacional de Saúde, 2016). A divulgação do programa incluiu palestras para as equipes técnicas das instituições de acolhimento da rede estadual, bem como anúncios/convites em redes sociais, como o Facebook. Os interessados que responderam ao convite para participar do programa foram alocados, conforme sua disponibilidade, nos dois grupos abertos.

Os encontros ocorreram de outubro a dezembro de 2018, no Instituto de Psicologia da Universidade Federal do Rio Grande do Sul em função de sua localização central e do fácil acesso. No primeiro encontro os participantes foram solicitados a preencher o Termo de Consentimento Livre e Esclarecido e as escalas para avaliação de eficácia do programa (T1). Após a 
conclusão da última sessão do programa, os participantes responderam à Ficha de Avaliação do Programa e, novamente, às escalas (T2). O uso de observadores treinados ao longo dos encontros favorece o aprimoramento do programa para estudos de replicação futuros, com maior controle sobre possível viés metodológico e maior credibilidade dos dados (Levitt et al., 2018).

\section{Resultados}

\subsection{Resultados quantitativos}

Foi realizada uma análise estatística descritiva das variáveis definidas como critérios para avaliação da implementação do programa, observando-se frequências de evasão e conclusão do programa pelos participantes, assim como amplitudes, médias e desvios-padrão da avaliação do moderador pelos participantes e observadores, satisfação dos participantes com o programa e compreensão/generalização dos conteúdos.

Quanto à Aceitabilidade e Adesão: dos 15 participantes selecionados para o estudo de viabilidade, 12 concluíram o programa, evidenciando sua aceitabilidade diante da proposta de intervenção. Três educadoras tiveram problemas de saúde ao longo da intervenção e interromperam sua participação (taxa de evasão=20\%). Considerando aqueles que concluíram o programa $(n=12)$, a frequência nos encontros foi superior a $80 \%$, possibilitando a emissão do certificado de participação.

Quanto à Avaliação do Moderador pelos Observadores, foram calculados os escores em relação às Habilidades Sociais (cinco itens) e à Integridade/Fidelidade (10 itens) do moderador. O escore relativo à avaliação do moderador foi calculado a partir da média dos escores atribuídos pelos observadores nas oito sessões, variando entre 1=Fez pouco e 4=Fez completamente. Os escores dos itens relativos às Habilidades Sociais variaram entre 1 e $4(M=3.91 ; D P=.21)$, e para Integridade/fidelidade de 1 a $4(M=3.83 ; D P=.24)$.

No que se refere à Satisfação dos Participantes com o Programa e com o Moderador, a variação dos escores obtidos em escala de autorrelato de quatro pontos (1-Insatisfeito; 2-Regular; 3-Satisfeito; 4-Muito satisfeito), preenchida após finalização do programa (T2), para as seguintes questões, foram: "Em geral, como se sentiu durante o programa?" escores entre 3 e 4 ( $M=3.67 ; D P=.49)$; "Qual a sua satisfação global com o programa?" escores entre 3 a $4(M=3.75 ; D P=.45)$; "Qual a sua avaliação do coordenador do grupo?" escores entre 3 a $4(M=3.92 ; D P=.28)$; "Qual a sua satisfação com as aprendizagens no programa?" escores entre 3 a $4(M=3.75 ; D P=.45)$; "Qual a sua satisfação com o tempo de duração das sessões?" escores entre 3 a $4(M=3.5 ; D P=.52)$; “Qual a sua satisfação com o horário das sessões?' escores entre 2 a 4 ( $M=$ 3.42; $D P=.66)$;

Com relação à Compreensão/Generalização dos Conteúdos pelos Participantes, os escores variaram entre 3 e 4 para todos os itens (1-Nada; 2-Muito Pouco; 3-Bastante; 4-Quase tudo), "Foi fácil assistir a todas as sessões do programa?"( $M=3.5$; $D P=.52)$; "Entendeu os conteúdos abordados durante as sessões?" $(M=3.5 ; D P=.52)$, e "Quanto você aplicou na vida cotidiana os conteúdos abordados no programa?" (M=3.33; $D P=.49)$.

\subsection{Resultados qualitativos}

Também foi realizada uma avaliação qualitativa do processo de implementação, considerando os registros dos observadores, as avaliações dos participantes e o diário de campo do moderador. Para isso foi utilizada uma Análise de Conteúdo, seguindo os passos propostos por Bardin (1977/2004): (1) Pré-análise: Organização do material, delimitação de um objetivo e hipóteses; (2) Descrição analítica: Estudo aprofundado do material descritivo e posterior categorização dos dados; (3) Interpretação referencial: Análise qualitativa dos dados, de modo a ampliar as reflexões sobre o assunto e atribuir significados aos resultados. Os dados foram organizados em três categorias temáticas definidas a posteriori: 1) resultados percebidos; 2) dificuldades encontradas; e 3) mudanças sugeridas. Além disso, foi utilizada triangulação por "método de coleta de dados", ou seja, diferentes meios de coleta (observações, diário de campo, questionários e autorrelatos dos participantes) e 
triangulação por "fonte de coleta dos dados", ou seja, dados coletados por diferentes pessoas (percepções dos participantes, observadores e do moderador), e em diferentes momentos - antes, durante e após o programa. A estratégia de triangulação de dados amplia a validade interna e o rigor científico de pesquisas qualitativas (Santos, Ribeiro, Queiroga, Silva, \& Ferreira, 2018).

Quanto ao eixo 1, que se refere aos resultados percebidos, os participantes apontaram, nas Fichas de Avaliação de Satisfação, estarem mais conscientes, reflexivos e autocríticos em relação a suas práticas educativas, destacando a tolerância, a paciência e a flexibilidade como aspectos que melhoraram após a participação na intervenção; um sentimento de maior amparo com o Programa Cuida; e maior disposição a buscarem suporte dos colegas e da equipe técnica quando necessário. O encontro sobre "comunicação assertiva" ganhou destaque na avaliação dos participantes, reverberando positivamente nas estratégias implementadas junto aos acolhidos. Ao serem solicitados a compartilharem como estavam utilizando os conteúdos trabalhados na prática cotidiana, mencionaram: "Usando uma comunicação mais clara e afetiva para ser assertiva", "Mais diálogo com os acolhidos", "Elogiando mais".

O moderador, a partir do seu diário de campo, e os observadores, a partir das fichas de avaliação, deram destaque ao encontro sobre "autocuidado", já que os educadores relataram com frequência se sentirem desamparados e pouco cuidados. Foi importante refletirem de forma coletiva sobre como o autocuidado é uma construção subjetiva e sobre a necessidade de flexibilizarem suas metas e estratégias de autocuidado, inclusive solicitando auxílio profissional quando fosse preciso. Alguns educadores se emocionaram nas dinâmicas "fale de mim para mim" e de "relaxamento conduzido", relatando a sensação positiva, porém delicada, de olhar para si mesmo, já que essa não era uma prática frequente.

No diário de campo do moderador destacaram-se, também, as iniciativas individuais utilizadas pelos educadores para superar o desamparo institucional. Uma das educadoras sociais relatou que produzia "scrapbooks" para cada acolhido que se encontrava em processo de desligamento, ou seja, confeccionava artesanalmente um álbum personalizado de memórias do acolhido para dar à família que o acolheria posteriormente. O compartilhamento de iniciativas como esta permite que o conhecimento circule e o saber não fique restrito à equipe técnica e diretiva das instituições, sendo esta última uma reclamação frequente dos educadores ao longo dos encontros. Além disso, a oportunidade de trocar experiências e práticas realizadas no contexto laboral mostrou-se como uma forma de valorização dos profissionais.

Quanto ao eixo 2, sobre as dificuldades encontradas, destaca-se como principal aspecto, identificado pelo moderador e pelos observadores, a dificuldade de cumprimento do cronograma de atividades previamente estabelecido no período de duas horas. A oferta de um espaço de fala imparcial e colaborativo fazia com que os educadores se sentissem convidados a narrarem suas histórias e dividirem suas angústias, preenchendo, desta forma, a maior parte do tempo do encontro. Embora o descontentamento com o cumprimento do horário não tenha sido apontado pelos participantes na etapa descritiva das fichas de satisfação, no último encontro, quando foi realizada uma avaliação coletiva do Programa Cuida, esse aspecto foi mencionado como um ponto a melhorar.

$\mathrm{O}$ eixo 3 refere-se às mudanças sugeridas pelos participantes ou vistas como necessárias pelo moderador e observadores. Os educadores sugeriram a maior divulgação do Programa, a sua continuidade através de novos módulos e temáticas, bem como a possibilidade de realização em outros locais e turnos do dia. Também sugeriram mais encontros com a temáticas de autocuidado, o que vem ao encontro do destaque dado à temática pelos observadores e moderador no eixo 1.

Os observadores identificaram a necessidade de revisão na estrutura das fichas de observadores, bem como de alguns de seus itens. O moderador, em conformidade com as dificuldades evidenciadas no eixo 2, sugeriu a remoção de atividades das sessões para o cumprimento do tempo de duas horas pré-estabelecido. Estes dados foram discutidos a partir de reuniões da equipe de pesquisa, buscando-se avaliar a necessidade de mudanças na proposta. Assim, foram introduzidas mudanças na estrutura do programa e em instrumentos de avaliação, que estão descritas no Quadro 1. 
Quadro 1. Mudanças introduzidas no Programa Cuida a partir do estudo de viabilidade.

\begin{tabular}{|l|l|}
\hline Proposta inicial & Proposta final \\
\hline $\begin{array}{l}\text { Utilização de vídeos introdutórios elaborados pela } \\
\text { pesquisadora }\end{array}$ & $\begin{array}{l}\text { Retirada dos vídeos introdutórios, tendo em vista que } \\
\text { ocupavam tempo e repetiam conteúdos trabalhados nos } \\
\text { slides subsequentes }\end{array}$ \\
\hline $\begin{array}{l}\text { Ficha dos observadores: opções de resposta "não fez"; } \\
\text { "fez pouco"; "fez de modo quase completo" e "fez } \\
\text { completamente" }\end{array}$ & Inclusão de uma opção de resposta: "Não se aplica" \\
\hline $\begin{array}{l}\text { Ficha dos observadores: três perguntas descritivas ao } \\
\text { final }\end{array}$ & $\begin{array}{l}\text { Retirada das perguntas descritivas e inclusão de tópicos } \\
\text { para os observadores escreverem mais livremente }\end{array}$ \\
\hline $\begin{array}{l}\text { Ficha dos observadores: } 13 \text { itens sobre Procedimento; } 5 \\
\text { itens sobre Habilidades sociais do moderador; } 9 \text { itens } \\
\text { sobre Integridade/Fidelidade do moderador; }\end{array}$ & $\begin{array}{l}\text { Retirada dos itens 13 (sobre entrega das normas por } \\
\text { escrito) e 24 (sobre efetivação de técnicas cognitivo- } \\
\text { comportamentais) }\end{array}$ \\
\hline $\begin{array}{l}\text { Envio de lembretes individuais com data e horário do } \\
\text { próximo encontro; }\end{array}$ & $\begin{array}{l}\text { Organização de grupos no Whatsapp (com autorização } \\
\text { dos participantes) para lembretes coletivos com } \\
\text { data/horário do próximo encontro e fotos da técnica da } \\
\text { semana }\end{array}$ \\
\hline $\begin{array}{l}\text { Role-play: encenação e reencenação de situaçães } \\
\text { cotidianas do acolhimento }\end{array}$ & $\begin{array}{l}\text { Em função do tempo, só é possível realizar a encenação e } \\
\text { uma breve discussão sobre a situação encenada }\end{array}$ \\
\hline
\end{tabular}

Fonte: Autores.

É possível visualizar mudanças pontuais, como aquelas realizadas nas fichas dos observadores, mas também, alterações estruturais, como a exclusão de atividades ou procedimentos para adequar-se ao tempo de duas horas por encontro. Atenta-se, assim, para a relevância de estudos de viabilidade prévios aos estudos de eficácia de intervenções.

\section{Discussão}

O objetivo deste estudo foi a avaliação de viabilidade do Programa Cuida para a promoção de habilidades/capacidades educativas positivas em educadores sociais de instituições de acolhimento. Os resultados obtidos foram satisfatórios quanto aos diferentes critérios de viabilidade avaliados.

Quanto à aceitabilidade e adesão, a taxa de retenção observada, ou seja, de não-desistência, foi de $80 \%$. A taxa é considerada satisfatória, uma vez que dados na literatura sobre adesão ao tratamento e retenção de participantes, em modelos de programas de saúde conduzidos em contexto internacional, variam entre $20-30 \%$ podendo chegar a $50 \%$ de perda de participantes (Teague et al., 2018).

Considera-se que foram fatores facilitadores da aceitabilidade e adesão o apoio e endosso do órgão gestor estadual das instituições de acolhimento, a oferta de certificados emitidos pela universidade, bem como o fato da procura ser ativa, ou seja, os próprios educadores entravam em contato com a pesquisadora, garantindo, assim, o interesse genuíno. Esta taxa de aceitabilidade/adesão converge, também, com as altas pontuações registradas pelos participantes quanto à sua satisfação com o programa e com o moderador, bem como, com a compreensão e generalização dos conteúdos abordados nos encontros. Os itens "Qual a sua satisfação global com o programa?", "Qual a sua avaliação do coordenador do grupo?” e "Qual a sua satisfação com as aprendizagens no programa?" obtiveram as médias mais altas na avaliação dos participantes. A avaliação positiva do moderador, conforme relatos dos participantes registrados no diário de campo, relaciona-se ao seu conhecimento 
teórico e técnico sobre a temática, já que, além da atuação na área acadêmica, o moderador tinha experiência prévia como psicólogo em uma instituição de acolhimento.

Além da apreciação dos educadores sociais, o moderador também foi avaliado por dois observadores a partir das Fichas de Avaliação preenchidas em todos os encontros. Os resultados indicam que o moderador desenvolveu quase completamente ou completamente a maioria dos aspectos relacionados às habilidades sociais e à integridade/fidelidade ao programa.

Ademais, outro possível fator relacionado à adesão e à satisfação dos educadores foi o uso de um formato "multicomponentes" que consiste na combinação de diferentes abordagens e técnicas (psicoeducação, atividades individuais, dinâmicas grupais, tarefas de casa e técnicas de manejo do estresse, como o relaxamento) para trabalhar conteúdos diversificados relacionados à temática central, neste caso, as práticas educativas. A introdução de uma intervenção multimétodos é preferível, já que oferece um repertório mais variado de abordagens e procura atender às diferenças individuais e culturais ao longo do processo de aprendizagem (Czerka \& Mechlińska-Pauli, 2009). Outras intervenções realizadas no Brasil (Durgante \& Dell'Aglio, 2019) e no exterior (Schotanus-Dijkstra et al., 2017) vêm apontando benefícios do uso do formato "multicomponentes".

A dificuldade do cumprimento do tempo de duas horas por encontro, previamente estabelecido, foi apontada pelo moderador em seu Diário de Campo, pelos observadores nas Fichas de Avaliação e por alguns participantes nos relatos descritivos e nas Fichas de Avaliação de Satisfação, de modo que os itens "Qual a sua satisfação com o tempo de duração das sessões?" e "Qual a sua satisfação com o horário das sessões?" obtiveram as médias mais baixas. Desta forma, a implementação de algumas atividades do cronograma tiveram que ser repensadas, como, por exemplo, os vídeos introdutórios elaborados pela pesquisadora e o segundo momento do Role-play em que, após as discussões coletivas e sugestões dos outros participantes, os educadores deveriam reencenar situações cotidianas. As discussões em grupo seguiram sendo realizadas após a primeira encenação a fim de que pudessem refletir sobre as estratégias empregadas. Embora tenha-se cogitado aumentar o número de encontros, concluiu-se que a extensa carga de trabalho dos educadores sociais poderia dificultar sua adesão ao Programa Cuida. Outras intervenções realizadas junto a educadores sociais têm utilizado carga horária similar (Bersch, 2017; Prada, 2007), o que levou à manutenção do formato de oito sessões com frequência semanal do programa.

Por fim, a partir da demanda dos observadores, houve algumas adaptações e remoções de itens da ficha de avaliação das sessões, de modo a reduzir julgamentos ambíguos quanto aos critérios avaliados e permitir uma escrita livre a respeito de outros aspectos observados. Também, a partir dos relatos dos participantes, optou-se pela criação de grupos de Whatsapp para envio de lembretes sobre os encontros e sobre as técnicas da semana.

A articulação entre os dados coletados só foi possível a partir da dupla triangulação no delineamento da pesquisa, a partir do uso de diferentes métodos e fontes para a coleta de dados. De acordo com Miles e Huberman (1994) a validade interna em pesquisa qualitativa diz respeito aos dados transparecerem aquilo que, de fato, está sendo avaliado, desta forma, o método de triangulação na coleta de dados é fundamental, reduzindo possíveis vieses no processo de coleta de dados (Tobin \& Begley, 2004). Assim, a triangulação de dados permitiu que as diferentes fontes de dados dialogassem entre si, trazendo maior rigor metodológico para a coleta e análise.

\section{Considerações Finais}

Os estudos de viabilidade possibilitam que se faça ajustes e correções no modelo de intervenção proposta, fornecendo subsídios para a otimização de resultados. O presente estudo auxiliou na constatação de demanda emergente de melhoria/ampliação das práticas educativas positivas de educadores sociais de instituições de acolhimento, indicando a 
urgência da implementação de programas que ofertem novas ferramentas de trabalho, considerando a perspectiva da Parentalidade Positiva e da Metodologia Experiencial.

Embora os educadores sociais desempenhem um papel central no desenvolvimento e na educação de crianças e adolescentes acolhidos, são observados poucos trabalhos de intervenção direcionados a essa população, fazendo com que se sintam, frequentemente, desassistidos. Além de capacitá-lo continuamente para desenvolver de maneira positiva sua função de cuidado e proteção, é necessário, conforme Bersch, Yunes, Garcia e Piske (2018), que o educador social seja sensibilizado acerca de sua implicação e relevância neste processo de acolhimento de crianças e adolescentes.

Os dados obtidos neste estudo são promissores no sentido de exemplificar a importância de se conduzir intervenções que visem, sobretudo, lançar um olhar atento a estes profissionais, ofertando espaços de reflexão e construções coletivas, valorizando as práticas educativas positivas que já utilizam. Ainda que muitos estudos evidenciem a importância da formação e da capacitação dos profissionais das instituições (Barros \& Naiff, 2015; Halpern, Leite, \& Moraes, 2015; Guerra \& Del Prette, 2018), poucos apresentam propostas de intervenção com rigor empírico na condução e avaliações de resultados obtidos para amostras e contextos específicos.

Considera-se que a participação de apenas um educador do sexo masculino é uma das limitações da amostra. Embora as mulheres sejam a maioria dos profissionais nesta função, configurando o que chama-se de "feminização do cuidado" (Wendt \& Dell'Aglio, 2020), é possível que uma maior divulgação do Programa Cuida possa atingir de forma mais ampla a população masculina de educadores sociais. Sugere-se que estudos futuros contem com diferentes meios de divulgação (rede sociais, folder impresso, divulgação presencial) e que ampliem a oferta do programa para outras instituições, possibilitando, assim, a obtenção de amostras maiores.

A partir dos resultados obtidos neste estudo de viabilidade, sugere-se prosseguir com estudo de eficácia da intervenção, incluindo as adaptações sugeridas, bem como maior tamanho amostral para a constatação de efeitos da intervenção em variáveis de interesse junto ao público-alvo.

\section{Referências}

Avoglia, H. R. C., Silva, A. M., \& Mattos, P. M. (2012). Educador social: Imagem e relações com crianças em situação de acolhimento institucional. Revista Mal-Estar e Subjetividade, 12(1-2), 265-292. http://pepsic.bvsalud.org/scielo.php?script=sci_abstract\&pid=S1518-61482012000100010\&lng=pt\&nrm=iso

Bardin, L. (1977/2004). Análise de conteúdo (3a ed). Edições.

Barros, N. S. \& Naiff, L. A. M. (2015). Capacitação para educadores de abrigo de crianças e adolescentes: Identificando representações sociais. Estudos $e$ Pesquisas em Psicologia, 15(1), 240-259. 10.12957/epp.2015.16073

Bersch, A. A. S. (2017). Resiliência profissional e a Educação Ambiental: Promoção de ambientes de desenvolvimento em instituição de acolhimento. Tese (Doutorado) - Universidade Federal do Rio Grande, FURG. Rio Grande, Rio Grande do Sul. https://sistemas.furg.br/sistemas/as b/arquivos/bdtd/0000011532.pdf

Bersch A. A. S., Piske, E. L., Yunes, M. A. M, Garcia, N. M., Silveira, S. B. S., \& Pietro, A. T. (2019). Programa de formação de educadores sociais na promoção da resiliência profissional (e-book). Editora da FURG.

Bersch, A. A. S., Yunes, M. A. M., Garcia, N. M., \& Piske, E. L. (2018). Educador social promotor de boas práticas e resiliência em instituições de acolhimento. In L. I. C. Cavalcante, C. M. C. Magalhães, L. S. Corrêa, E. F. Costa, \& D. A. Cruz (Orgs). Acolhimento institucional de crianças e adolescentes: Teorias e evidências científicas para boas práticas. (pp 251-264).

Biscouto, K. D. (2012). Avaliação de um programa de intervenção em práticas educativas parentais para mães sociais. Dissertação (Mestrado). Universidade Estadual de Londrina. Londrina, Paraná. http://www.uel.br/pos/pgac/wp-content/uploads/2014/03/Avalia\%C3\%A7\%C3\%A3o-de-um-programa-deinterven\%C3\%A7\%C3\%A3o-em-pr\%C3\%A1ticas-educativas-parentais-para-m\%C3\%A3es-sociais.pdf

Bowen, D. J., Kreuter, M., Spring, B., Cofta-woerpel, L., Linnan, L., Weiner, D., \& Fernandez, M. (2009). How we design feasibility studies. American Journal of Preventive Medicine, 36(5), 452-457. 10.1016/j.amepre.2009.02.002

Chen, Y., Haines, J., Charlton, B. M, \& VanderWeele, T. J. (2019). Positive parenting improves multiple aspects of health and well-being in young adulthood. Nature Human Behaviour, 3(7), 684-691. 10.1038/s41562-019-0602-x

Conselho Nacional de Justiça, CNJ (2020). Diagnóstico sobre o Sistema Nacional de Adoção e Acolhimento. https://www.neca.org.br/wpcontent/uploads/SNA_Relatorio_Diagnostico-Sistema-Nacional-Adocao-Acolhimento_2020.pdf 
Conselho Nacional de Saúde (2016). Resolução $n^{\circ}$ 510/2016. Dispõe sobre a pesquisa em Ciências Humanas e Sociais. Brasil: Ministério da Saúde, http://conselho.saude.gov.br/resolucoes/2016/Reso510.pdf

Council of Europe (2006). Recommendation Rec(2006)19 of The Committee of Ministers to Member States on Policy to Support Positive Parenting. (Adopted by the Committee of Ministers on 13 December 2006 at the 983rd meeting of the Ministers' Deputies). https://search.coe.int/cm/Pages/result_details.aspx?ObjectID=09000016805d6dda.

Creswell, J. W. (2003). Qualitative, quantitative and mixed methods approaches. Sage Publications.

Czerka, E. \& Mechlińska-Pauli, M. (2009). Teaching and learning in different cultures: An adult education perspective. Gdańsk: Gdańsk Higher School of Humanities Press.

Durgante, H. (2019). Desenvolvimento, implementação e avaliação do Programa Vem Ser: Programa de psicologia positiva para a promoção de saúde de aposentados. Tese (Doutorado) - Universidade Federal do Rio Grande do Sul, UFRGS, https://lume.ufrgs.br/handle/10183/194393

Durgante, H. \& Dell'Aglio, D. D. (2018). Critérios metodológicos para a avaliação de programas de intervenção em Psicologia. Avaliação Psicológica, 17(1), 155-162. 10.15689/ap.2017.1701.15.13986

Durgante, H \& Dell'Aglio, D. D. (2019). Multicomponent positive psychology intervention for health promotion of Brazilian retirees: a quasi-experimental study. Psicologia: Reflexão e Crítica, 32(6), 1-14. 10.1186/s41155-019-0119-2

Gortner, E. M., Rude, S. S, \& Pennebaker, J. W. (2006). Benefits of expressive writing in lowering rumination and depressive symptoms. Behavior Therapy, 37(3), 292-303. 10.1016/j.beth.2006.01.004

Guerra, L. L. \& Del Prette, Z. P. (2018). Habilidades sociais educativas de cuidadores de crianças institucionalizadas. Arquivos Brasileiros de Psicologia, 70(3), 98-112. http://pepsic.bvsalud.org/scielo.php?script=sci_arttext\&pid=S1809-52672018000300008\&lng=pt\&tlng=pt.

Halpern, E. E., Leite, L. M. C., \& Moraes, M. C. M. B. (2015). Seleção, capacitação e formação da equipe de profissionais dos abrigos: O hiato entre o prescrito e o real. Trabalho, Educação e Saúde, 13, Supl. 1, 91-113. 10.1590/1981-7746-sip00033

Kappler, S. R. \& Mendes, D. M. L. F. (2019). Trocas Afetivas de Crianças em Acolhimento Institucional. Psicologia: Ciência e Profissão, 39 , e184527. https://doi.org/10.1590/1982-3703003184527

Levitt, H. M., Bamberg, M., Creswell, J. W., Frost, D. M., Josselson, R., \& Suárez-Orozco, C. (2018). Journal article reporting standards for qualitative primary, qualitative meta-analytic, and mixed methods research in psychology: The APA Publications and Communications Board task force report. American Psychologist, 73(1), 26-46. 10.1037/amp0000151

Máiquez, M. L., Rodrigo, M. J., Capote, C., \& Vermaes, I. (2000). Aprender en la vida cotidiana: Un programa para padres. Visor.

Martín-Quintana, J. C., Máiquez-Chavez, M. L., Rodrigo-López, M. J., Byme, S., Rodríguez-Ruiz, B., \& Rodríguez-Suárez, G. (2009). Programas de Educación Parental. Psychosocial Intervention, 18(2), 121-133. http://scielo.isciii.es/scielo.php?script=sci_arttext\&pid=S113205592009000200004\&lng=es\&tlng=es

Martínez-González, R. A. (2009). Programa-Guía para el Desarollo de Competencias Emocionales, Educativas y Parentales. Madrid: Ministerio de Sanidad y Política Social.

Marzol, R. M., Bonafé, L., \& Yunes, M. A. M. (2012). As perspectivas de crianças e adolescentes em situação de acolhimento sobre os cuidadores protetores. Revista Psico, 43(3), 317-324. http://revistaseletronicas.pucrs.br/ojs/index.php/revistapsico/article/viewFile/7988/8232

Miles, M. B., \& Huberman, A. M. (1994). Qualitative data analysis: an expanded source book. (2a ed.), Sage Publication.

Moré, C. L. O. O. \& Sperancetta, A. (2010). Práticas de pais sociais em instituições de acolhimento de crianças e adolescentes. Psicologia \& Sociedade, 22(3), 519-528. doi: 10.1590/S0102-71822010000300012

National Institute For Health Research - NIHR. (2017). Feasibility and pilot studies: Which programme should I apply to? http://www.nets.nihr.ac.uk/glossary.

Prada, C. G. (2007). Avaliação de um programa de práticas educativas para monitoras de um abrigo infantil. Tese (Doutorado) - Universidade Federal de São Carlos, UFSCar, São Carlos, São Paulo. https://repositorio.ufscar.br/bitstream/handle/ufscar/2828/TeseCGP.pdf?sequence=1\&isAllowed=y

Martín-Quintana, J. C. M. \& Rodrigo-López, M. J. R. (2013). La promoción de la Parentalidad Positiva. Revista Educação, Ciência e Cultura, 18(1), 77-88. $10.18316 / 1090$

Rodrigo, M. J. (2016). Quality of implementation in evidence-based positive parenting programs in Spain: Introduction to the special issue. Psychosocial Intervention, 25(2), 63-68. 10.1016/j.psi.2016.02.004

Rodrigo-López, M. J., Máiquez-Chavez, M. L., \& Martín-Quintana, J. C. (2010). La educación parental como recurso psicoeducativo para promover la parentalidad positiva. FEMP.

Romero, D. L., Akiba, H. T., Dias, A. M., \& Serafim, A. P. (2016). Transtornos mentais comuns em educadores sociais. Jornal Brasileiro de Psiquiatria [online], 65(4), 322-329. 10.1590/0047-2085000000140.

Santos, K. S., Ribeiro, M. C., Queiroga, D. E. U., Silva, I. A. P., \& Ferreira, S. M. S. (2018). O uso de triangulação múltipla como estratégia de validação em um estudo qualitativo. Ciência e Saúde Coletiva, 25(2), 655-664. 10.1590/1413-81232020252.12302018 
Research, Society and Development, v. 10, n. 9, e52710918412, 2021

(CC BY 4.0) | ISSN 2525-3409 | DOI: http://dx.doi.org/10.33448/rsd-v10i9.18412

Schotanus-Dijkstra, M., Pieterse, M. E., Drossaert, C. H. C., Walburg, J. A., \& Bohlmeijer, E. T. (2017). Possible mechanisms in a multicomponent email guided positive psychology intervention to improve mental well-being, anxiety and depression: A multiple mediation model. Journal of Positive Psychology, 14(2), 141-155. 10.1080/17439760.2017.1388430

Schuler. E. \& Dias, C. M. S. B. (2014). Avós que criam seus netos: Uma proposta de intervenção psicoeducativa. Investigação Qualitativa em Saúde, 2, 134139. https://proceedings.ciaiq.org/index.php/CIAIQ/article/view/509/504

Silva, J. (2009). Parents Raising Safe Kids: ACT 8-Week Program for Parents. American Psychological Association.

Silveira, M. A. S., Maruschi, M. C., \& Bazon, M. R. (2012). Risco e proteção para o engajamento de adolescentes em práticas de atos infracionais. Revista Brasileira de Crescimento e Desenvolvimento Humano, 22(3), 348-357. 10.7322/jhgd.46699

Teague, S., Youssef, G. J., Macdonald, J., Sciberras, E., Shatte, A., Fuller-Tyszkiewicz, M., \& Hutchinson, D. (2018). Retention strategies in longitudinal cohort studies: A systematic review and meta-analysis. 10.31234/osf.io/fzk2w

Tobin, G. A. \& Begley, C. M. (2004). Methodological rigour within a qualitative framework. Journal of Advanced Nursing, 48(4), 388-396. 10.1111/j.13652648.2004.03207.x

Wendt, B. \& Dell’Aglio, D. D. (2020). A 'feminização' do cuidado nos contextos de acolhimento institucional In: C. M. C. Magalhães, L. I. C. Cavalcanti, A. M. J. Silva, \& E. J. S. Cruz, Aspectos peculiares do acolhimento institucional de crianças e adolescentes (pp.149-160). Editora: Appris Editora

Wendt \& Dell'Aglio (submetido). Escala de Competências Educativas, Emocionais e Sociais do Educador Social - ECEES: Construção e investigação das propriedades psicométricas.

Yamaoka, Y. \& Bard, D. E. (2019). Positive parenting matters in the face of early adversity. American Journal of Preventive Medicine, 56(4), 530-539. 10.1016/j.amepre.2018.11.018 